\title{
Estressee e estilo parental materno no transtorno de déficit de atenção e hiperatividade
}

\author{
Joseana Azevedo Bargas \\ Pontifícia Universidade Católica de Campinas \\ Marilda Emmanuel Novaes Lipp \\ Pontifícia Universidade Católica de Campinas
}

\begin{abstract}
Resumo
Este estudo avaliou a influência do estresse e do estilo parental materno sobre o estresse dos filhos avaliados pela instituição onde os dados foram coletados como portadores de TDA/H. Participaram 25 mães e os respectivos filhos, estes com idade média de 9,4 anos. As crianças foram avaliadas pela Escala de Estresse Infantil, e as mães, pelo Inventário de Sintomas de Estresse para Adulto de Lipp e Inventário de Estilos Parentais de Gomide. Os resultados mostraram que esta população apresenta alto nível de estresse (estilo parental classificado como de risco), que a sintomatologia do estresse materno tem relação com o subtipo de TDA/H e que e os sintomas de desatenção são potencializados pelo estresse. Não foi encontrada relação significativa entre estresse materno e infantil.

Palavras-chave: Stress, transtorno da falta de atenção com hiperatividade, estilo parental.
\end{abstract}

\section{Stress and maternal parental style in attention deficit hyperactivity disorder}

\begin{abstract}
In this study we evaluate the influence of stress and of maternal parenting style on the stress level of children evaluated by institution where data were collected as bearers of ADHD. The participants were 25 mothers and their children (average age 9.4 years). The children were assessed by the Children Stress Scale and their mothers by Lipp Stress Symptoms inventory for Adult and with the Gomide Parenting Styles Inventory . The results show that this population presents a high level of stress, parental style classified as at-risk. It also revealed that the symptomatology of maternal stress is related to the subtype of ADHD and that symptoms of inattention are potentiated by excessive stress. No significant relationship between maternal stress and childhood stress was found.
\end{abstract}

Key words: Stress, attention deficit disorder with hyperactivity, parenting style.

\section{Estrés y estilo parental materno en el trastorno de déficit de atención e hiperactividad}

\section{Resumen}

Este estudio evaluó la influencia del estrés y del estilo parental materno sobre el estrés de los niños evaluados por la institución donde se recolectaron datos de portadores de TDA/H. Participaron 25 madres y sus respectivos hijos, estos con edad promedio de 9,4 años. Se evaluó a los niños con la Escala de Estrés Infantil y a las madres con el Inventario de Síntomas de Estrés para Adulto de Lipp e Inventario de Estilos Parentales de Gomide. Los resultados mostraron que este grupo presenta alto nivel de estrés (estilo parental clasificado como de riesgo), que la sintomatología del estrés materno tiene relación con el subtipo de TDA/H y que los síntomas de falta de atención son potencializados por estrés. No se encontró relación significativa entre estrés materno e infantil.

Palabras Clave: Estrés, trastorno de falta de atención con hiperactividad, estilo parental. 


\section{Introdução}

As várias fases de desenvolvimento intelectual, emocional e afetivo trazem consigo a oportunidade de o indivíduo desenvolver seu potencial genético, porém essas etapas são marcadas por múltiplas situações geradoras de tensão, muitas vezes incapacitantes para as crianças e para o seu frágil mecanismo de combate ao estresse (Maturano, 2008). Os Transtornos de desenvolvimento constituem uma ameaça potencial para o aparecimento de problemas emocionais, comportamentais e estresse. Um dos principais transtornos neuropsicológico da infância é o TDA/H - Transtorno de Déficit de Atenção e Hiperatividade, cuja incidência é estimada em $3 \%$ a $5 \%$ entre as crianças em idade escolar no Brasil (Benzick, 2000). Estas crianças apresentam dificuldades de adaptação ao meio em que vivem, nem sempre conseguindo corresponder às expectativas dos adultos, e muitas vezes são descritas como preguiçosas, mal-educadas, desobedientes e inconvenientes. O impacto causado atinge a criança e todas as pessoas envolvidas com ela, como sua família e a escola. Geralmente os pais sentem-se perdidos no manejo com essas crianças, necessitando de intervenções que os capacitem para essa ação. O modo como os pais irão relacionar-se com seus filhos faz acentuada diferença no prognóstico do quadro e também no nível de estresse da díade mãe-filho (Bignotto, 2000). Como já observaram Johnston e Mash (2001), compreender a influência das práticas parentais no desenvolvimento das crianças com TDA/H pode fornecer uma informação significativa quanto ao tipo de intervenção a ser realizado para promover uma boa adaptação dessas crianças. Há muito já se tem ciência de quanto o estilo parental influencia o desenvolvimento e a consolidação das funções de regulação, no geral. Nos casos de TDAH, em que essas funções são frequentemente prejudicadas, ainda mais importante se torna os pais desenvolverem práticas que venham a colaborar para um melhor funcionamento de seus filhos (Darling \& Steinberg, 1993). Diversas pesquisas buscam analisar a influência de práticas parentais no funcionamento de crianças com TDA/H. Campbell (2002) pesquisou como o processo de socialização na família pode contribuir para o surgimento, agravamento e manutenção de comportamentos caracterizados como TDA/H. Adicionalmente, um estudo realizado por Ellis e Nigg (2009) indicou correlação entre inconsistência na disciplina materna e o tipo combinado de TDA/H, e também com todos os tipos de comportamento dos filhos com TDA/H (transtorno desafiador opositivo, conduta de desordem, desatenção e hiperatividade-impulsividade). A conclusão foi que aspectos específicos da parentalidade estão relacionados ao TDA/H, independentemente da classificação dos comportamentos. Conforme observaram Ellis e Nigg (2009), as pesquisas na área indicam que a falta de estratégias dos pais e estilos parentais deficitários parecem se relacionar com a exacerbação de sintomas de TDA/H. A esta conclusão chegou também Kunrath, (2006), cuja pesquisa revelou que, em termos gerais, os pais, estressados pela dificuldade referente à educação de seus filhos, utilizavam- -se demais de estratégias coercitivas do que indutivas, o que apontava a necessidade de orientar os pais dessas crianças no sentido de colocar em prática métodos mais eficazes de educar os filhos (Kunrath, 2006). Adicionalmente, a pesquisa de Ramos-Quiroga, Vidal e Casas (2013) mostrou a necessidade de uma visão multiprofissional na educação e tratamento de crianças com TDA/H no tocante à educação parental. Pesquisas adicionais são necessárias para identificar se de fato a falta de estratégias de coping dos pais se relaciona com o estresse dos filhos e se contribui para o agravamento dos sintomas de TDA/H.

\section{O estresse}

De acordo com Lipp (1996), o estresse é uma reação do organismo com componentes físicos e/ou psicológicos, causada pelas alterações psicofisiológicas que ocorrem quando a pessoa se confronta com uma situação amedrontadora ou que a faça muito feliz. Esta reação produz desequilíbrio no funcionamento global do ser humano, e quando excessiva, enfraquece seu sistema imunológico, deixando-o sujeito a infecções e doenças. O processo de estresse se desenvolve em quadro fases: alerta, resistência, quase-exaustão e exaustão (Lipp, 2001).

O desenvolvimento infantil gera mudanças físicas, cognitivas, emocionais, afetivas e sociais. Segundo Lipp (2000a), as mudanças inerentes ao desenvolvimento levam as crianças a enfrentar situações de estresse. O processo de estresse pode desencadear-se quando a criança não possui repertório de enfrentamento para lidar com as demandas do meio (Lipp, 2008). Os transtornos de desenvolvimento constituem uma ameaça potencial para o aparecimento de problemas emocionais e comportamentais e de estresse, e um desses transtornos neuropsicológicos da infância é o TDA/H (Rohde, 2003). No caso especifico deste transtorno, a literatura indica uma interação entre a presença de TDA/H na criança e o estresse materno. Yousefia, Far e Abdolahian (2011) verificaram em uma pesquisa que mães de filhos com TDA/H possuem um nível de estresse mais elevado e tendem a utilizar estilo parental mais coercitivo e punitivo do que as mães cujos filhos não têm esse transtorno. Os autores concluíram que o nível de estresse afeta a escolha do modo de tratar os filhos com TDA/H, e que o uso de punição e disciplina errática das mães pode levar a surgirem ou se exacerbarem nos filhos comportamentos agressivos ou de oposição. Os resultados levam à conclusão de que o comportamento negativo dos pais em relação aos filhos com TDA/H tem relação com níveis elevados de cortisol e de estresse, e também com comportamentos de oposição. Altos níveis de crítica parental suscitaram uma maior resposta do cortisol. Isto ressalta a importância da interação entre pais e filhos para a prevenção de problemas de comportamento.

Segundo Lipp e Romano (1987), a criança reage com sensações físicas e psicológicas quando se vê diante de um evento estressor. Os distúrbios psicológicos mais comuns são depressão, enurese, dificuldades de relaciona- 
mento, comportamento agressivo, desobediência inusitada, ansiedade, choro excessivo, gagueira, terror noturno, dificuldades escolares, pesadelos, introversão súbita e insônia. Os distúrbios físicos são asma, dores de cabeça, tique nervoso, dores abdominais, diarreia, náusea, hiperatividade, ranger de dentes, tensão muscular, distúrbio de apetite, enurese noturna, gagueira, doenças dermatológicas e outros. No caso da criança portadora de TDA/H, os sintomas de estresse se somam às dificuldades típicas do quadro de TDA/H.

\section{O Transtorno de Déficit de Atenção e Hiperatividade - TDA/H}

O TDA/H é uma síndrome heterogênea, de etiologia multifatorial, dependendo de fatores genéticos e adversidades biológicas e psicossociais. A literatura na área aponta alguns dos fatores psicossociais que poderiam estar relacionados ao desenvolvimento ou agravamento do transtorno, entre eles, aspectos específicos da parentalidade como inconsistência na disciplina maternal, conforme sugerem Ellis e Nigg (2009), além de alto nível de estresse das mães, apontado por Yousefia e cols. (2011). Adicionalmente, Campbell (2002) sugere que o processo de socialização na família pode contribuir para o surgimento, agravamento e manutenção de comportamentos característicos do TDA/H. Taylor e Sonuga-Barke (2008) encontraram em sua pesquisa uma relação significativa entre expressão negativa de emoções dos pais e TDA/H nos filhos.

Segundo Cypel (2007), é muito complexa a discussão sobre as causas implicadas na determinação da desatenção e hiperatividade, e o mais provável é que uma somatória de fatores desencadeantes origine o distúrbio atencional e hiperatividade. $\mathrm{O}$ diagnóstico do TDA/H sustenta-se sobre dois pilares: a) os dados da história clínica da criança; b) avaliações neuropediátricas e neuropsicológicas. O DSM-IV(1994) define este transtorno como um problema de saúde mental, considerando-o um distúrbio bidimensional, que envolve a atenção e a hiperatividade/impulsividade. A característica essencial é um padrão persistente de desatenção e/ou hiperatividade, mais frequente e severo do que aquele tipicamente observado em crianças de mesma idade que estejam em nível equivalente de desenvolvimento. Seu diagnóstico é baseado em sintomas descritos no DSM-IV, que classifica estes pacientes em três tipos: a) TDA/H com predomínio de sintomas de desatenção; b) TDA/H com predomínio de sintomas de hiperatividade/impulsividade; c) TDA/H combinado.

Segundo Barkley (1997), o déficit mais importante no $\mathrm{TDA} / \mathrm{H}$, independentemente da hiperatividade e da desatenção, é um defeito na inibição da conduta, o que tem grande impacto no funcionamento cotidiano (pragmático), nas praxias do desempenho escolar, social, etc. Como praxias o autor entende a memória de trabalho (verbal e não verbal), o controle do afeto, a motivação, o estado de alerta e tudo o que afete o autocontrole e a capacidade de conduta dirigida para metas.
A orientação terapêutica para um prognóstico favorável ao desenvolvimento dessa criança envolve um trabalho que deve ser realizado junto à criança, à família e à escola através de psicoeducação, e tem sido indicada como necessária a terapia cognitiva comportamental, associada ou não ao uso de medicações (Cypel, 2007).

\section{Relação entre pais e filhos}

A educação dos filhos sempre foi uma tarefa complexa para os pais, e quando a criança tem TDA/H é, sem dúvida, uma tarefa mais dificultosa e perturbadora (estímulo estressor) para qualquer família. A relação familiar é o fator mais importante em termos de prognóstico, da forma como se expressam os sintomas e dos problemas que lhe são associados. A família pode ter o papel de proteção, contenção e apoio, mas pode também ser um elemento potencializador de dificuldades e de estresse dos filhos.

Segundo Costa, Teixeira e Gomes (2000) e Gomide (2003), os pais, buscando que seus filhos sigam princípios morais e desenvolvam independência, autonomia e responsabilidade, direcionam o comportamento deles no sentido de que possam exercer adequadamente o papel social quando jovens e adultos. As estratégias e técnicas utilizadas pelos pais para orientar o comportamento dos filhos são denominadas práticas educativas parentais, e o conjunto dessas práticas denomina-se Estilo Parental (Gomide, 2003), que é o modo como os pais gerenciam as questões de poder, hierarquia e apoio emocional no relacionamento com os filhos. Atualmente na literatura não se encontram estudos sobre os estilos parentais empregados por pais de crianças com TDA/H.

Gomide (2006) considera como práticas educativas positivas a Monitoria positiva e o Comportamento moral

A Monitoria positiva corresponde a comportamentos parentais de atenção às atividades e à adaptação dos filhos (regras claras, frequência equilibrada da atenção, demonstração segura do afeto, acompanhamento e supervisão das atividades escolares e de lazer).

O Comportamento moral, obtido pelo exemplo dos pais, refere-se ao desenvolvimento do senso de justiça, empatia e responsabilidade, generosidade e do conhecimento do certo e do errado.

As práticas educativas negativas o abuso físico e psicológico, o relaxamento da disciplina, a monitoria negativa (supervisão estressante), a negligência e a punição inconsistente.

O abuso físico e psicológico é caracterizado pela imposição de disciplina com base em práticas corporais negativas, que são consideradas um fator de risco para o desenvolvimento de problemas sociais e psicológicos (condutas antissociais e distúrbios psiquiátricos). O abuso psicológico caracteriza-se principalmente pela ameaça, chantagem de abandono e humilhação da criança. O desenvolvimento da autonomia e das relações sociais é prejudicado, podendo causar baixa autoestima e depressão. O estudo de Manso (2004) apontou a importância de fatores econômicos e cultu- 
rais no nível de estresse na família que se reflete no método de educar os filhos. O autor encontrou que quanto mais intenso o nível de estresse familiar maior era a ocorrência de violência contra a criança.

O relaxamento da disciplina se caracteriza pelo relaxamento das regras estabelecidas, e é considerado um fator de risco para os problemas de conduta e delinquência. As regras são estabelecidas, os pais ameaçam, e quando se deparam com comportamentos de oposição dos filhos, abrem mão de seu papel educativo.

A monitoria negativa ou supervisão estressante se traduz pelo excesso de instruções e regras, não importando o seu cumprimento, e torna hostil o ambiente familiar.

A negligência se caracteriza pela falta de atenção e afeto. Os pais negligentes não são responsivos, retiram-se de situações difíceis e ignoram a maioria dos comportamentos e atividades das crianças. Sentimentos de insegurança, vulnerabilidade e dificuldade nos relacionamentos sociais acabam sendo gerados por esta negligência.

A punição Inconsistente é um tipo de punição que interfere sobretudo na percepção que a criança desenvolve sobre si, pois, ao utilizarem essa prática, os pais se orientam pelo seu humor no momento de punir ou reforçar, e não pelo ato praticado pela criança.

O estilo parental de risco para o desenvolvimento de comportamentos antissociais pelas crianças é caracterizado pela utilização, por parte pais, de práticas parentais consideradas negativas, como. por exemplo, negligência, punição inconsistente e abuso físico, em detrimento das positivas. Ao contrário, o uso das práticas consideradas positivas (comportamento moral e monitoria negativa) em vez das negativas caracteriza um estilo parental ótimo (Gomide, 2006).

Segundo Gomide (2006), os estilos parentais exercem influência no desenvolvimento de características pessoais das crianças e adolescentes. Tais características são determinantes para a adaptação da criança às diversas mudanças (biológica, social e cognitiva) com que se depara nesse período da vida. Desse modo, é possível compreender a influência dos estilos parentais no nível de estresse apresentado pelas crianças e adolescentes com TDA/H, já que a relação familiar é um fator importante em termos de prognóstico, da forma como se expressam os sintomas e dos problemas que the são associados.

Acredita-se que, no contexto do TDA/H, os pais menos estressados capacitem seus filhos para lidar melhor com os fatores estressores da vida, ou seja, estas crianças seriam menos estressadas, pois receberiam uma criação que proporciona autoestima, autoconfiança, habilidades sociais, entre outras características positivas. Um estudo que mostra a relação entre níveis de estresse, prática parental e comportamentos integrantes do TDA/H é o publicado por Christiansen, Oades, Psychogiou, Hauffa e Sonuga-Barke (2010). Esses autores avaliaram o nível de cortisol salivar de crianças com e sem o TDA/H durante sessões experimentais eliciadoras de estresse emocional, nas quais os pais faziam ou três elogios ou três críticas aos filhos na presença deles. Os resultados indicaram que crianças com $\mathrm{TDA} / \mathrm{H}$ foram mais sensíveis ao estresse representado pelas críticas e comentários negativos dos pais do que as outras. Especificamente, comportamentos de oposição sofreram aumento na presença de críticas e reclamações de pais que já haviam sido avaliados pelos filhos como muito críticos.

O Centro Nacional de Recursos sobre o TDA/H (2013) reconhece que alguns estilos parentais podem aumentar os sintomas de hiperatividade, desatenção e impulsividade em crianças com déficit de atenção e hiperatividade, porém o centro afirma que os estilos parentais podem não ser a única causa do TDA/H. A literatura existente aborda inúmeras influências que o estilo parental pode exercer sobre o desenvolvimento da criança, mas pesquisas que visem relacionar o estilo parental com TDA/H e estresse são poucas. Entre as existentes encontra-se a pesquisa de Yousefia e cols. (2011). Este trabalho visou comparar estresse entre as mães com crianças de TDA/H e mães de crianças normais. O estudo incluiu crianças de cinco a doze anos de idade que tinham sido encaminhadas a clínicas de psiquiatria infantil. Os resultados mostraram que houve diferença significativa entre o nível de estresse de mães de crianças com TDA/H e mães de crianças normais. Houve uma diferença significativa também de estilos parentais entre filhos de mães de TDA/H e mães de crianças sem o transtorno. Assim, quanto maior o estresse dos pais, mais arbitrário e errático será o estilo parental. Estes dados confirmam os de autores anteriores, tais como os de Anastopoulos, Guevremont, Shelton e Dupaul (1992); Baker, (1994); Baldwin, Brown e Milan (1995); Barkley, Fischer, Edelbrock e Smallish (1990); Beck, Young e Tarnowski (1990); Breen e Barcley (1988); Pisterman, Fireston e MC Garth (1992); Harrison e Sofronoff (2002); Jennifer (2010); Johnston e Mash (2001); Mash e Johnston (1983) e Wood (2007) Esses pesquisadores verificaram alta relação entre a existência de filhos com TDA/H e estresse familiar. Há ainda o trabalho, já mencionado, de Christiansen e cols. (2010), que concluíram, com base em seus dados, que o comportamento negativo dos pais para com os filhos com TDA/H tem relação com níveis elevados de cortisol e de estresse dos filhos, e também com comportamentos de oposição. Altos níveis de crítica parental suscitaram uma maior resposta do cortisol. Adicionalmente, Bignotto (2010) também encontrou uma associação significativa entre o estresse materno e o dos filhos, em um estudo que verificou a eficácia do treino de controle do estresse infantil.

Embora esses estudos mostrem associações entre estilo parental, estresse e TDA/H, ainda não existem dados que permitam afirmar com certeza a existência desta associação. O presente trabalho teve por objetivo aprofundar o conhecimento sobre a relação entre o estilo parental, o estresse e o TDA/H em uma amostra de crianças. 


\section{Método}

\section{Participantes}

O desenvolvimento deste estudo teve a participação de 25 crianças com diagnóstico de TDA/H, com idades variando de 7 a 13 anos, de ambos os sexos, que cursavam escolas da rede particular e municipal do Interior do Estado de São Paulo e de suas respectivas mães. Todas as crianças estavam em atendimento no Projeto Casulo - "Centro de Atenção à Aprendizagem e ao Comportamento Infantil". Foram excluídas as que possuíam diagnóstico de intelectualmente deficiente (QI inferior a 70), conforme avaliação prévia feita pela instituição, ou distúrbio psiquiátrico grave. Foram excluídas as mães que possuíam distúrbio psiquiátrico grave previamente diagnosticado.

Desse modo, a amostra ficou composta por 25 mães e seus filhos, estes com a média de idade de 9,4 anos (DP=1.47), sendo $84 \%$ do sexo masculino e $16 \%$ do sexo feminino. A hipótese diagnóstica foi levantada pela instituição. Dentre as crianças, $20 \%$ eram do subtipo de TDA/H desatento, $20 \%$ eram crianças hiperativas e $60 \%$ eram do subtipo combinado.

\section{Material}

Após a assinatura do Termo de Consentimento Livre e Esclarecido e o preenchimento da ficha de identificação das mães (data de nascimento, idade, profissão, estado civil, escolaridade), procedeu-se à coleta de dados utilizando-se: a)- o ISSL - Inventário de Sintomas de estresse para Adultos (Lipp, 2000b), que avaliou a presença ou não de estresse, os tipos de sintomas físicos e psicológicos apresentados e a fase do estresse em que as mães se encontravam: alerta, resistência, quase exaustão e exaustão; b)- a ESI - Escala de Estresse Infantil (Lipp \& Lucarelli, 1997), instrumento que tem por objetivo verificar a presença de estresse em crianças de seis a catorze anos de idade, de ambos os sexos, constituindo-se de 35 itens em escala Likert de zero a quatro pontos, relacionados a sintomas físicos, psicológicos, psicológicos com componentes depressivos e psicofisiológicos de estresse; e c)- Inventário de Estilos Parentais (Gomide, 2006), em que existe um questionário específico para mães, um para os pais e um para os filhos. O IEP contempla 42 situações que avaliam sete variáveis (duas práticas educativas positivas e cinco negativas). Os escores do IEP revelam o estilo parental adotado pelos pais (pai e mãe separadamente), que pode ser: estilo parental ótimo; estilo parental regular, acima da média; estilo parental regular, abaixo da média; e estilo parental de risco.

No presente trabalho os estilos foram classificados como estilo parental bom, estilo parental regular (abaixo da média, indicando a importância de os pais participarem de grupos de treinamento de pais) e estilo parental de risco para o desenvolvimento de comportamentos antissociais.

\section{Procedimento}

Foi realizada uma reunião com a equipe multidisciplinar do Projeto Casulo, a fim de definir o objetivo e a metodologia da pesquisa e fazer o levantamento das crianças e das mães que preenchiam o critério de inclusão para participar do estudo. As crianças selecionadas já tinham o diagnóstico de TDA/H feito por avaliações neuropsicológicas, neurológicas, fonoaudiológicas e pedagógicas.

As mães das crianças selecionadas foram convidadas a participar de um encontro que ocorreu na instituição. Todas as mães que compareceram à reunião aceitaram participar da pesquisa, assinaram o Termo de Consentimento Livre e Esclarecido e preencheram a ficha de identificação. A coleta de dados foi feita na instituição com a aplicação do Inventário de Sintomas de Estresse para Adultos e do Inventário de Estilos parentais materno.

As crianças selecionadas foram convidadas a participar da pesquisa. A aplicação da Escala de Estresse Infantil ocorreu individualmente nos dias em que elas se submetiam a intervenções na instituição

\section{Método de análise dos dados}

Na comparação das variáveis categóricas entre os grupos com e sem estresse e entre os tipos de transtorno TDA/H foi utilizado o teste Qui-Quadrado de Pearson, ou o teste exato de Fisher, com valores esperados menores que 5. Para comparar as variáveis contínuas entre os grupos com e sem estresse foi utilizado o teste de Mann-Whitney, devido à ausência de distribuição normal das variáveis; e na comparação entre os tipos de transtorno TDA/H e a faixa etária da criança, foi utilizado o teste Kruskal-Wallis. O nível de significância adotado para os testes estatísticos foi de $5 \%$.

\section{Resultados e discussão}

os resultados indicaram um alto índice de estresse nesta amostra, pois $72 \%$ das mães e também das crianças apresentaram sintomas de tensão excessiva. Dentre as mães com estresse, $60 \%$ estavam na fase de resistência, que é a fase intermediária do processo do estresse, com a prevalência de $72 \%$ de sintomas psicológicos. Estes dados confirmam o trabalho de Yousefia e cols. (2011), que apontaram o fato de mães de crianças com TDA/H mostrarem tendência a experimentar um alto nível de estresse, cuja consequência direta foi a de afetar o estilo parental que elas adotam e, por conseguinte, o nível de estresse dos filhos.

Dentre as crianças, $36 \%$ estavam na fase de resistência e $47 \%$ apresentavam predominância dos sintomas psicológicos. Estes resultados confirmam achados de outras pesquisas, que mostram que pais mais estressados tendem a ter filhos com mais sintomas de estresse (Bignotto, 2010; Manso, 2010). Segundo Lazarus (1966) e Ellis (1973), as diversas formas de apoio social que as crianças recebem, 
principalmente o dos pais, podem influenciar os fatores de vulnerabilidade ao estresse infantil. Estes achados receberam confirmação no estudo de Christiansen e cols. (2010), que encontraram aumentos de cortisol salivar em crianças nos momentos de desafio, quando os pais as criticavam ou demostravam emoções negativas em relação ao desempenho dos filhos.

Pesquisas mostram que pais de crianças com TDA/H, quando comparados com pais de crianças sem diagnóstico psiquiátrico, relatam maior nível de estresse ligado ao manejo da criança e maior discordância entre os cônjuges a respeito das práticas educativas (Campbell 2000). Campbell (2002) enfatizou que o processo de socialização na família pode contribuir para o surgimento, agravamento e manutenção de comportamentos característicos de TDA/H. Segundo Rohde e Matos (2003), diversos conflitos nas relações familiares são gerados quando surgem sintomas de dificuldades de atenção, hiperatividade e impulsividade. Esta visão é também partilhada por outros autores, como Ellis e Nigg (2009), em cujo entendimento aspectos específicos da parentalidade como, por exemplo, inconsistência na disciplina maternal, estão relacionados ao TDA/H independentemente da classificação dos comportamentos.

Como postula Ellis (1973), provavelmente não são os acontecimentos em si o que provoca as emoções, mas a maneira como eles são interpretados. Deste modo, acredita-se que as mães têm grande dificuldade em entender e aceitar as dificuldades de seus filhos, interpretando, de forma errada, a incompetência como desobediência a exercer algumas atividades, o que potencializa tanto o seu estresse como o estresse nas crianças. Os dados indicam ser necessário que as mães aprendam a distinção entre desobediência e incompetência (dificuldade). Sabe-se que a maioria dos comportamentos da criança com TDA/H ocorrem em razão de suas dificuldades, e não de teimosia. Quando aprendem a fazer esta distinção as mães conseguem reduzir punições inadequadas e orientar a criança para desenvolver comportamentos mais assertivos, promovendo um desenvolvimento favorável de suas potencialidades (Benzick, 2000).

No presente estudo verificou-se que o maior número de crianças com sintomas de estresse era do subtipo de TDAH combinado, seguido pelas crianças hiperativas, e que o estresse parece atingir menos as crianças do subtipo desatento. Encontrou-se associação significativa entre subtipo de TDA/H e tipo de sintoma de estresse materno $(p=0009)$, indicando que a desatenção é um estímulo menos estressante para as mães com filhos desatentos, as quais apresentavam um grau menor de sintomas de estresse de natureza psicológica. Todas as crianças desatentas tinham mães com sintomas psicológicos, enquanto que $80 \%$ das crianças hiperativas possuíam mães com sintomas físicos e 90\% das crianças com TDA/H combinado tinham mães com sintomas psicológicos, conforme mostra a tabela 1.

Possivelmente isto se deve ao fato de que crianças com hiperatividade motora sofrem um desgaste físico maior, o que poderia potencializar os sintomas físicos.

Outro resultado importante refere-se à intensidade dos sintomas do estresse infantil. Com base nos dados referentes às respostas dadas na questão 3 "Tenho dificuldade de prestar atenção" da Escala de Estresse Infantil, observou-se que $71,43 \%$ das crianças que não tinham estresse afirmavam sentir apenas "um pouco" de dificuldades atencionais, enquanto $72,22 \%$ das crianças com estresse indicaram sentir "sempre" dificuldades atencionais. Como se pode ver na tabela 2 , esta diferença é significativa ( $p=$ 0.001 ), ou seja, existe uma diferenciação entre as crianças com e sem sintomas de estresse na percepção da intensidade do sintoma de desatenção.

Tabela 1. Comparação entre tipos de sintomas ISSL e subtipo de TDA/H.

\begin{tabular}{llll}
\hline Sintomas ISSL & Desatento & Hiperativo & Combinado \\
\hline Psicológico & $100,00 \%$ & $20,00 \%$ & $90,91 \%$ \\
\hline Físico & 0 & $80,00 \%$ & $9,09 \%$ \\
\hline
\end{tabular}

Teste Exato De Fisher: $p=0.009$

Tabela 2. Relação entre dificuldades atencionais e estresse infantil.

\begin{tabular}{lll}
\hline Dificuldade atencional & Crianças s/ estresse & Crianças c/ estresse \\
\hline Um pouco & $71,43 \%$ & 0 \\
\hline Bom & $28,57 \%$ & $11,11 \%$ \\
\hline Regular & 0 & $16,67 \%$ \\
\hline De risco & 0 & $72,22 \%$ \\
\hline
\end{tabular}

Teste exato de Fisher: $p=0.001$ 
Este achado pode ser de utilidade, uma vez que abre caminho para futuras intervenções que objetivem o treino do controle do estresse, o que tornaria possível diminuir os sintomas de desatenção de uma maneira não medicamentosa. Este conhecimento poderá ter consequências para as áreas de reabilitação cognitiva, educacional, familiar e social. Atualmente a reabilitação cognitiva para o TDA/H é feita com base na técnica de treino de função cognitiva, objetivando a neuroplasticidade, técnica de compensação de função cognitiva que busca compensar os déficits com a utilização de apoios externos, orientações psicoeducativas para a família e a escola, e terapia cognitivo-comportamental. O treino do controle de estresse seria mais uma ferramenta para a reabilitação na busca de auxiliar o desenvolvimento destas crianças desatentas e melhorar sua qualidade de vida. $\mathrm{Na}$ área educacional, a diminuição dos sintomas de desatenção poderia facilitar o processo de ensino e aprendizagem, uma vez que a criança estaria mais atenta e um maior número de informações seria codificado, armazenado e posteriormente evocado, podendo ajudar na diminuição do fracasso escolar. Por isso é importante uma intervenção para instrumentalizar as crianças a lidar com este estresse e possivelmente diminuir este sintoma de desatenção

O teste de Kruskal-Wallis revelou não haver diferença significativa quando se comparam o tipo de TDA/H e a idade da criança $(p=0.533)$.

Quanto à classificação do estilo parental, houve predomínio do estilo parental de risco, seguido pelos estilos regular, bom e ótimo, sendo que $81 \%$ das mães que possuíam estilo parental de risco também apresentavam sintomas de estresse, resultado que independe do subtipo de TDA/H. Constatou-se também que o número de crianças com estresse aumenta à medida que diminui a utilização, por parte das mães, de práticas parentais consideradas positivas. Durante a coleta de dados foi possível mais uma vez verificar a desorientação das mães no tocante tanto ao manejo do estresse como a estratégias assertivas para conduzir a educação de seus filhos, mostrando grande dificuldade de adaptação. Esta realidade é preocupante, já que o prognóstico favorável do TDA/H depende muito da relação entre a criança e sua mãe, pois quando os estilos parentais são adequados, auxiliam no enfrentamento dos problemas e favorecem o desenvolvimento da criança.

\section{Conclusão}

Inúmeros autores têm sugerido, com base em suas pesquisas, que os pais de crianças com TDA/H necessitam, como prioridade para o desenvolvimento adequado dessas crianças, aprender a utilizar práticas parentais na forma de estratégias educativas que demonstrem aceitação e compreensão das dificuldades envolvidas no transtorno e que envolvam o apoio emocional de seus filhos (Darling \& Steinberg, 1993; Johnston \& Mash, 2001; Kunrath, 2006). Ramos-Quiroga e cols. (2013), adicionalmente, enfatizam a necessidade de um tratamento muldimodal para promover uma boa adaptação dessas crianças

Conclui-se que crianças com TDA/H e suas mães apresentam alto nível de estresse, estilo parental materno classificado como de risco para o desenvolvimento de comportamentos antissociais e que a sintomatologia do estresse materno tem relação com o subtipo de TDA/H. Nesta amostra também se concluiu que a desatenção é um estimulo menos estressante que a hiperatividade, sendo que os sintomas de desatenção são potencializados pela tensão excessiva. Desse modo acredita-se que programas que instrumentalizem as mães e as crianças a manejarem o estresse podem trazer grandes benefícios para a qualidade de vida destas pessoas, através da minimização dos sintomas de desatenção e utilização de práticas parentais positivas.

Embora a amostra utilizada tenha sido pequena, espera-se que os dados desta pesquisa possam contribuir, mesmo que modestamente, para uma melhor compreensão do nível de estresse envolvendo esta população e um meIhor entendimento do impacto dos estilos parentais no nível de estresse dos filhos, para melhor elucidação quanto aos efeitos do subtipo de TDA/H da criança na natureza da sintomatologia materna. Consequente e adicionalmente, também se espera que educadores e outros profissionais que lidam com essa população se conscientizem da capacidade do estresse infantil de potencializar os sintomas de desatenção na criança portadora de TDA/H e, consequentemente, de que a desatenção pode ser reduzida promovendo-se um ambiente menos estressante em casa e na escola.

Como recomendam vários autores, entre eles Fisher (1990); Kunrath, (2006); Ramos-Quiroga e cols. (2013), o tratamento do TDA/H envolve uma abordagem multifocal, englobando intervenções psicossociais e psicofarmacológicas. As pesquisas indicam a necessidade de apoio para os pais, potencializando os recursos existentes dentro dessas famílias, para que todos possam melhor lidar com as dificuldades inerentes ao TDA/H.

Assim sendo, pesquisadores devem continuar a estudar esta questão, assim como avaliar novas situações e, ainda, propor a elaboração de estratégias que se construam de formas cada vez mais eficazes de auxiliar na tarefa de educar os filhos, principalmente os portadores de TDA/H. Os dados desta pesquisa, assim como os de outras anteriores encontrados na literatura, levam ainda a questionar se o estresse leva a um estilo parental de risco ou o estilo parental de risco levaria ao estresse. Para responder a esta questão se fazem necessárias pesquisas com um número maior de participantes e que estas avaliem outros fatores envolvidos no estresse dos pais. Importante também é averiguar se os comportamentos envolvidos no TDA/H podem ser aliviados com a mudança de práticas parentais negativas por meio da educação dos pais. Se isto for cientificamente comprovado, deverão ser objeto de tratamento não só os portadores do transtorno, mas também seus pais. 


\section{Referências}

American Psychiatric Association (1994). Diagnostic and statistical manual of mental disorders (4a ed.). Washington, DC: Author.

Anastopoulos, A. D., Guevremont, D. C., Shelton, T. L., \& Dupaul, G. J. (1992). Parenting estresse among families of children with attention deficit hyperactivity disorder. Journal of Abnormal Child Psychology, 20(5), 503-520.

Baldwin, K., Brown, R. T., \& Milan, M. A. (1995). Predictors of estresse in caregivers of attention deficit hyperactivity disordered children. The American Journal of Family Therapy, 23(2), 149-160.

Baker, D. B. (1994). Parenting estresse and ADHD: A comparison of mothers and fathers. Journal of Emotional \& Behavioral Disorders, 2(1), 46-51.

Barkley, R. A. (1997). ADHD and the nature of self-control. New York: Guilford Press.

Barkley, R. A., Fischer, M., Edelbrock, C., \& Smallish, L. (1990) The adolescent outcome of hyperactive children diagnosed by research criteria, I: An 8 year prospective follow-up study. Journal of the American Academy of child and adolescent psychiatry. 29 546-557.

Benczick, E. B. (2000). Transtorno de déficit de atenção/ hiperatividade: Atualização diagnóstica e terapêutica. São Paulo: Casa do Psicólogo.

Beck, S. J., Young, G. H., \& Tarnowski, K. G. (1990). Mental characteristics and perceptions of pervasive and situational hyperactive and normal controls. Journal of the American Academy of Child and Adolescent Psychiatry, 88, 150-156.

Breen, M. J., \& Barcley, R. A. (1988). Child psychopathology and parenting estresse in girls and boys having attention deficit disorde with hyperactivity. Journal of pediatric psychology, 13(2), 265-280.

Bignotto, M. M. (2000). O papel dos pais na prevenção do estresse infantil. Em M. N. Lipp (Org.), Crianças eestresseadas: causas, sintomas e soluções (pp. 101-121). Campinas, SP: Papirus.

Bignotto, M.M. (2010). A eficácia do treino de controle do estresse infantil. Tese de Doutorado em Psicologia, Pontifícia Universidade Católica de Campinas, Campinas-SP.

Campbell, S. B. (2000). Attention-deficithyperactivy disorder: a developmental view. Em A. J. Sameroff, M. Lewis \& S. M. Miller (Eds.), Handbook of developmental psychopathology (pp. 383401). New York: Kluwer/Plenum.

Campbell, S. B. (2002). Problemas de externalização em préescolares: questões clínicas e de desenvolvimento. New York: Guilford Press.
Costa, F. T., Teixeira, M. A., \& Gomes, W. B. (2000). Responsividade e exigência: duas escalas para avaliar estilos parentais. Psicologia: Reflexão e Crítica, 13(3), 465-473. Recuperado: 11 mai 2010. Disponível: http://www.scielo.br/scielo.php?script=sci_ arttext\&pid=S0102-79722000000300014\&lang=pt

Christiansen, H., Oades, R. D., Psychogiou, L., Hauffa, B. P., \& SonugaBarke, E. (2010). Does the cortisol response to estresse mediate the link between expressed emotion and oppositional behavior in Attention-Deficit/Hyperactivity-Disorder (ADHD)? Recuperado: 23 ago 2013. Disponível: http://www.behavioralandbrainfunctions. com/content/6/1/45

Cypel, S. (2007). Déficit de atenção e hiperatividade e as funções executivas: Atualização para pais, professores e profissionais da saúde. São Paulo: Lemos Editorial.

Darling, N.L. \& Steinberg, L. (1993). Parental style as a contex: an integrative model. Psych Bull, 113, 487-496.

Ellis, A. (1973). Humanistic Psychology: The rational emotive approach. New York: Julian.

Ellis, B., \& Nigg, J. (2009). Parenting Practices and Attention-Deficit/ Hyperactivity Disorder: Partial Specificity of Effects. J.Am.Acad Child Adolesc Psychiatry. 48(2), 146-154

Fischer, M. (1990). Parenting estresse and the child with attention deficit hyperactivity disorder. Journal of Clinical Child Psychology, 19(4), 337-346

Gomide, P.I. (2003). Estilos parentais e comportamento anti-social. Em A. Del Prette \& Z. A. P. Del Prette (Orgs.), Habilidades Sociais, Desenvolvimento e Aprendizagem: Questões conceituais, avaliação e intervenção. Campinas, SP: Alínea Editora.

Gomide, P. I. (2006). Inventário de estilos parentais - IEP: modelo teórico, manual de aplicação, apuração e interpretação. Petrópolis, RJ: Vozes.

Harrison, C., \& Sofronoff, K. (2002). ADHD and parental psychological diestresse: role of demographics, child behavioural characteristics, and parental cognitions. Journal of the American Academy of Child and Adolescent Psychiatry, 41(6), 302-312.

Jennifer, T. (2010). Predicting parenting estresse in families of children with $A D H D$ (Eds.). University of Toronto.

Johnston, C., \& Mash, E. J. (2001). Families of children with attentiondeficit/hyperactivity disorder: review and recommendations for future research. Clinic Child and family psychology review, 4(3), 183-207.

Kunrath, L. H. (2006). Estratégias educativas: a perspectiva de pais de crianças com transtorno de déficit de atenção/hiperatividade. Dissertação de Mestrado, Faculdade de Psicologia Social e da Personalidade, Pontifícia Universidade Católica do Rio Grande do 
Sul, Porto Alegre-RS.

Lazarus, R. S. (1966). Psychological estresse and the coping process. New York: McGrawHill.

Lipp, M. N. (1996). Estresse: Conceitos Básicos. Em M. N. Lipp (Org.), Pesquisas sobre estresse no Brasil: Saúde, ocupações e grupos de risco (pp. 17-31). Campinas, SP: Papirus.

Lipp, M. N. (2000a). O estresse da criança e suas consequências. Em M. N. Lipp (Org.), Crianças eestresseadas: causas, sintomas e soluções (pp. 101-121). Campinas, SP: Papirus.

Lipp, M. N. (2000b). Inventário de sintomas de estresse para adultos. São Paulo: Casa do Psicólogo.

Lipp, M. N. (2001). O Estresse e a Beleza da Mulher. São Paulo: Connection Books.

Lipp, M. N. (2008). Crianças Eestresseadas: Causas, sintomas e soluções (5a ed.). Campinas, SP: Papirus.

Lipp, M. N., \& Lucarelli, M. D. (1997). Escala de estresse infantil. São Paulo: Casa do Psicólogo.

Lipp, M. N., \& Romano, A. S. F. (1987). O estresse infantil. Estudos de Psicologia, 4, 42-54.

Manso, J. M. M. (2004). Influência del estrés familiar, desempleo y precariedad econômica em los malos tratos a la infância. Investig. Psicol. 9(2), 127-152.

Marturano, E. M., \& Gardinal, E. C. (2008). Um estudo prospectivo sobre o eestressee cotidiano na $1^{\text {a }}$ série. Aletheia, 27, 81-97.
Mash, E. J., \& Johnston, C. (1983). Parental perceptions of child behaviour problems, parenting self- esteem, and mothers reported estresse in younger and older hyperactive and normal children. Journal of Consulting and Clinical Psychology, 51, 1371-1381.

National Resource Center on ADHD. Parent Education and Training on ADHD. Recuperado: 23 ago 2013. Disponível: http://www. chadd.org/About-CHADD/National-Resource-Center.aspx

Pisterman, S., Fireston, P., \& Garth, M. C. (1992). The role of parent training in treatment of preschoolers with ADHD. American journal of Psychiatry, 62, 397-408.

Ramos-Quiroga, J. A., Vidal, R., \& Casas, M. (2013). Toward a Better Understanding of Attention-Deficit/Hyperactivity Disorder Across the Lifespan. Journal of the American Academy of Child \& Adolescent Psychiatry 52(2), 53-162

Rohde, L. A., \& Mattos, P. (2003). Princípios e práticas em transtorno de déficit de atenção/ hiperatividade. Porto Alegre: Artmed.

Taylor, E. A., \& Sonuga-Barke, E. J. S. (2008). Disorders of attention and activity. Em M. Rutter, D. Bishop, D. Pine, S. Scott, J.S. Stevenson, E. A. Taylor e cols. (Eds.), Rutter's Child \& Adolescent Psychiatry (5a ed., pp 521-542). U.K.: Wiley-Blackwell.

Yousefia, S., Far, A. S., \& Abdolahian, E. (2011). Parenting estresse and parenting styles in mothers of ADHD with mothers of normal children. Procedia - Social and Behavioral Sciences 30, 1666 1671

Wood, J. M. (2007). Examining estresse among parents of children with attention deficit Hyperactivity Disorder (Eds.). New York: University of Rochester.

\section{Sobre as autoras}

Joseana Azevedo Bargas (joseanaazevedo@hotmail.com) Mestre pela Pontifícia Universidade Católica de Campinas.

Correspondência: Rua José Martins Amorim, 112, apto 14B, Pq. Colinas da Mantiqueira, São João da Boa Vista, São Paulo, SP. CEP: 13874-370.

Marilda Emmanuel Novaes Lipp (marildalipp@puc-campinas.edu.br)

Professora Titular da Pontifícia Universidade Católica de Campinas.

Correspondência para Marilda Emmanuel Novaes Lipp: Pontifícia Universidade Católica de Campinas, Centro de Ciências da Vida - Programa de Pós Graduação em Psicologia. Av. John Boyd Dunlop s/nº - Prédio dos Ambulatórios de Especialidades. Jardim Ipaussurama, Campinas, SP. CEP: 13060-904

Este trabalho é resultado da dissertação de Metrado em Psicologia da primeira autora na PUC-Campinas. Para a sua realização, contou-se com o apoio financeiro da CNPQ na forma de bolsa para a primeira autora. 